\title{
Staphylococcus Aureus Malignant External Otitis
}

\author{
Filippo Ricciardiello1*, Michele Cavaliere1, Flavia Oliva², Annalisa Pianese1, \\ Teresa Abate ${ }^{1}$, Carlo Antonio Leone ${ }^{3}$ \\ ${ }^{1}$ Head and Neck Department, Otorhinolaryngology Unit, The University of Naples "Federico II", Naples, Italy \\ ${ }^{2}$ Otorhinolaryngology Unit, Cardarelli Hospital, Naples, Italy \\ ${ }^{3}$ Otorhinolaryngology Unit, Monaldi Hospital, Naples, Italy \\ Email: ${ }^{\text {filipporicciardiello@virgilio.it }}$
}

Received 1 July 2014; revised 2 August 2014; accepted 1 September 2014

Copyright (C) 2014 by authors and Scientific Research Publishing Inc.

This work is licensed under the Creative Commons Attribution International License (CC BY).

http://creativecommons.org/licenses/by/4.0/

c) (i) Open Access

\begin{abstract}
Malignant external otitis is a severe infection of the external auditoy canal, characterized by high gravity and mortality. It can arrive to skull base and originate intracranial complications. The most frequent pathogenic agent is Pseudomonas aeruginosa. Authors described two cases of external malignant otitis caused by Staphylococcus aureus, explaining clinical features, progression, diagnostic and therapeutic approach, prognosis of this disease. A comparison between Pseudomonas aeruginosa and Staphylococcus aureus malignant otitis was described, associated with a literature analysis.
\end{abstract}

\section{Keywords}

Malignant External Otitis, Necrotizing External Otitis, Skull Base, Facial Nerve Palsy, Cerebellar Abscesses

\section{Introduction}

Malignant external otitis (MEO) is a severe infection of the external auditoy canal (EAC). It is characterized by high gravity, fast evolution and high mortality (46\%). It is also called "necrotizing external otitis" because of the distructive nature of this infection.

It was first identified by Meltzer and Kelemen in 1959, then Chandler, in 1968, described its clinical details [1].

The causative agent most commonly implicated is Pseudomonas aeruginosa [2]-[5]. Other possible pathogens

*Corresponding author.

How to cite this paper: Ricciardiello, F., Cavaliere, M., Oliva, F., Pianese, A., Abate, T. and Leone, C.A. (2014) Staphylococcus Aureus Malignant External Otitis. Advances in Microbiology, 4, 878-885. http://dx.doi.org/10.4236/aim.2014.413097 
are: Staphylococcus aureus [2] [6], Staphylococcus epidermidis [7] [8], Proteus mirabilis [9], Klebsiella oxytoca [10] [11] and fungi [12], such as Aspergillus fumigatus [13].

Diabetes mellitus or other conditions that compromise immune system, such as acquired immunodeficiency syndrome, malignancy, chemotherapy could represent risk factors [2] [14] [15].

Trigger factors are usually traumas (often caused by common maneuvers to clean the external ear) or exposure to swimming pool water (with high halogens concentration).

Typical symptoms are: unremitting otalgia (worse during the night), otorrhea, aural fullness, hearing loss, headache, temporomandibular joint pain.

The infection starts from the ear skin and causes chondritis and osteomyelitis, extended posteriorly toward the mastoid, anteriorly into the temporo-mandibular joint, medially to the petrous bone apex or inferiorly (more often) to soft tissue of subtemporal area, through Santorini fissures.

The infection could extend to skull base and cause cranial nerve paralisis [16]. Because of its location in the temporal bone, facial nerve is usually the first involved [17]. Facial nerve damage can be caused by neurotoxic effect, compression or nervous fibers distruction. Facial involvement is a progression sign, but it is not linked to a worse prognosis [18].

Jugular foramen nerves are the second affected, after facial. Petrous apex involvement can cause abducens and trigeminal damage or, more medially, optic nerve damage. Sigmoid sinus or internal jugular vein trombosis, meningitis and cerebral abscess are other possible complications. Skull base osteomyelitis can spread to the controlateral side and include cervical spine.

MEO diagnosis is based on clinical presentation, laboratory tests, histology (especially for differential dignosis with ear carcinoma), imaging studies (CT/MRI) and nuclear imaging [19] [20].

MEO treatment includes long term systemic antibiotic therapy, local treatment, immunosuppression and metabolic control (especially diabetes mellitus) and surgery, in selected cases.

The aim of the authors is to describe two cases of Staphylococcus aureus MEO in order to discuss the clinical features and to emphasise the importance of a proper diagnostic and therapeutic procedures to improve the prognosis of a disease that has a mortality rate still high.

\section{Case Reports}

\subsection{Case 1}

In October 2006, a 57-year-old Italian woman was admitted to ENT Department of Cardarelli Hospital of Naples, with intense left ear pain and otorrhea, that she had treated with amoxicillin + clavulanic acid and nimesulide, headache, vertigo and vomiting. Patient had also diabetes mellitus type II, treated with oral hypoglycemic therapy, arterial hypertension, lower limbs vascular disease and frequent episodes of external auditory canal furuncles treated with topical antibiotics.

Physical examination revealed a large polypoid tissue at the osseous-cartilaginous junction in upper and posterior part of the external left ear canal and abundant purulent secretion; the tympanic membrane was intact.

We performed a microbiological culture and a biopsy of the polypoid tissue: the culture was positive for Staphylococcus aureus and the histopathological examination showed granulation with chronic inflammation without evidence of malignancy.

Computed tomography (CT) demonstrated soft-tissue edema of the external ear canal, canal bone and mastoid destruction, evidence of osteitis of the squama occipitalis with penetrating by the inflammatory process (Figure 1).

MRI brain showed the presence of two contiguous abscesses surrounded by edema with contrast agent irregular impregnation in the left cerebellar hemisphere, compression of the fourth ventricle and brain stem in the foramen magnum, engagement of the upper worm in the quadrigeminal cistern with compression of the midbrain with initial obstructive hydrocephalus (Figure 2, Figure 3).

Laboratory tests showed: hyperglycemia (326 mg/dl), HbA1c 10.9\% (suggestive for a long history of poorly controlled diabetes mellitus), ESR $45 \mathrm{sec}$, neutrophilic leukocytosis and hyperazotemia. Anti-nuclear antibodies (ANA) were elevated, without ANCA (anti-neutrophil cytoplasmic antibodies) presence. There was not releaved deficit either of umoral and cellular immunity.

Waiting for ear swab results, an empiric antibiotic therapy with ceftazidime (1 gr twice a day) and levofloxacin (500 mg twice a day) in parenteral somministration and insulin to regularise glycemic levels were administered. 


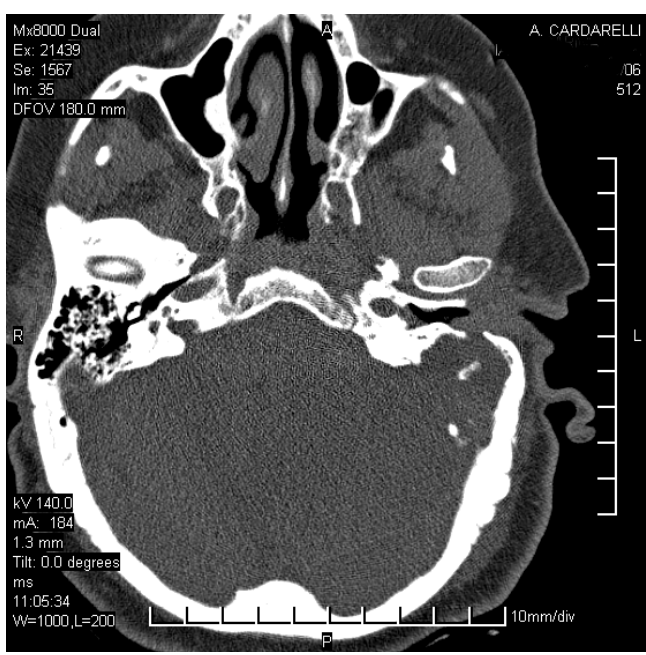

Figure 1. Canal bone and mastoid destruction.

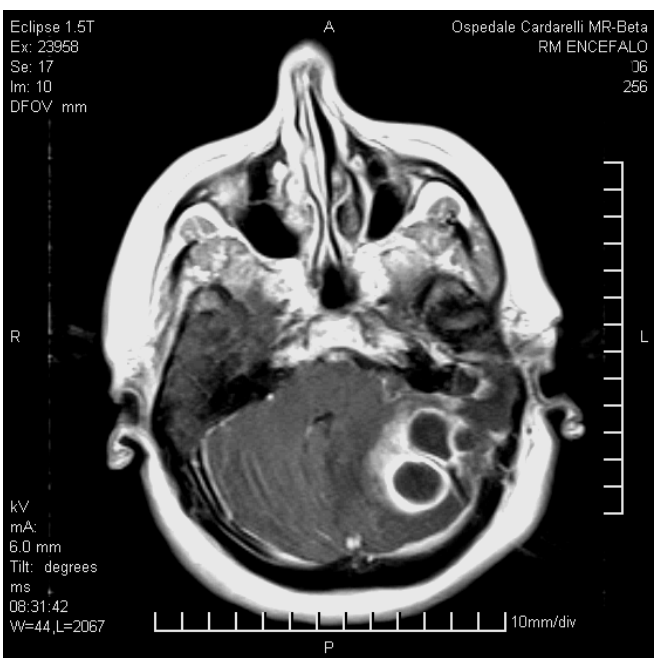

Figure 2. Two contiguous abscesses surrounded by edema in the left cerebellar hemisphere.

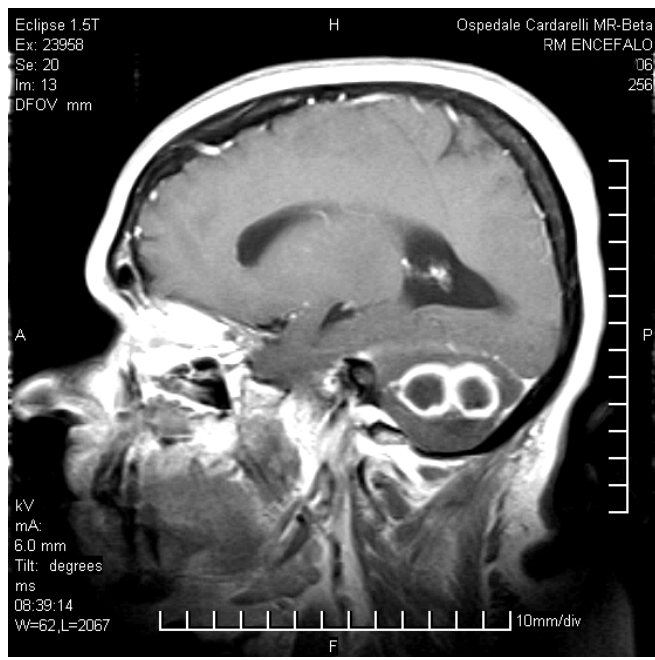

Figure 3. Abscesses in left cerebellar hemisphere. 
According to the bacterial cultural results, ceftazidime was changed with teicoplanin (400 mg/day).

Ear canal was medicated with solution of boric acid in alcohol at $70^{\circ}$ and through a curettage of granulations that occluded the canal.

After 7 days, brain MRI revealed persistence of cerebellar abscesses, with decrease of perilesional edema, re-expansion of the fourth ventricle, less decrease of brain stem in the foramen magnum and of upper worm in the quadrigeminal cistern (Figure 4).

The patient was submitted to neurosurgical intervention in order to drain the two cerebellar abscesses. Two weeks after operation, oto-neurologic symptoms regressed, persisting only slight edema of ear canal skin and pain only to acupressure. During the third week, ear swab resulted negative and there was a further improvement in symptoms. Antibiotic therapy was continued only with levofloxacin (active to Staphylococcus and Pseudomonas aeruginosa, responsible of a possible superinfection) to complete 8 weeks of therapy.

The patient was submitted to a multidisciplinary follow-up: ENT, neurological and neurosurgical.

\subsection{Case 2}

In January 2005, a 79-year-old Italian man affected by arterial hypertension, diabetes mellitus type 2, previous ischemic stroke, was admitted to ENT departement of University of Naples "Federico II", because of left otalgia, mucopurulent otorrhea, left temporo-parietal pain, vertigo from 20 days. The patient referred many episodes of external otitis over the years, treated with topic antibiotics. Last time he used amoxicillina per os and topic neomicin, without clinical improvement.

Physical examination revealed a granulation tissue on the posterior wall of left external canal and in its osseous-cartilaginous junction.

CT showed inflammatory tissue in left external ear canal, tympanic cavity and mastoid.

Laboratory tests revealed: neutrophilic leukocytosis, hyperglycemia (260 mg/dL), glycosuria, high ESR (55 $\mathrm{mm} / \mathrm{h})$.

So it was begun a parenteral therapy with ciprofloxacina $200 \mathrm{mg}$ (1fl twice a day) + ceftazidime $1 \mathrm{gr}$ ( $1 \mathrm{fl}$ twice a day), waiting for the ear swab. Ear canal was medicated with alcohol boric solution.

Ear swab pointed out Staphylococcus aureus meticillin-resistant (MR), and so ceftazidime was replaced with teicoplanin (200 mg twice a day). Following days swabs confirmed the causative agent. The auditory canal biopsy showed a fibro-inflammatory process with acute and xanto-granulomatous aspects.

After five days, the patient had a complete left facial nerve palsy and so he was undergone to an operation of mastoidectomy with successive clinical features improve, despite palsy persistence (third grade BH scale).

Antibiotic therapy duration was 6 weeks and teicoplanin was suspended 10 days after surgery.

Six months later, the patient has again otorrhea, intense otalgia, vertigo and left facial nerve palsy worsening (sixth grade BH scale). CT showed mastoidectomy outcomes on the left ear, soft tissue edema and a round focal alteration, in appearance pseudo-abscess, inferiorly to EAC; material of homogeneous tissue density on new cavum medial wall with second facial tract erosion (Figure 5). Therefore the patient was again submitted to ear swab, that reconfirmed Staphylococcus aureus, and to antibiotic therapy with teicoplanin and levofloxacin.

\subsection{Laboratory Exams Did Not Show a Dysmetabolism}

Mastoidectomy revision was performed, with a successive clinical improvement, disappearance of otalgia, otorrhea and vertigo and regression of palsy to second grade BH scale.

Antibiotic therapy was protracted up to 8 weeks (teicoplanin up to 15 days after surgery).

\section{Discussion}

Rare cases of MEO caused by Staphylococcus aureus are reported in literature [2] [6] and it is interesting to compare this infection with more frequent kind of MEO caused by Pseudomonas aeruginosa.

In Pseudomonas aeruginosa MEO facial palsy is generally early and frequent [16] [21] because it is subsequent to neurotoxins diffusion through stylomastoid foramen; Staphylococcus aureus MEO, instead, could cause only a direct nerve lesion, so it is less frequent and usually late-onset; in fact we observed a late-onset facial palsy only in the second case.

In MEO diagnosis it is important to examine and to integrate clinical anamnestic findings, microbiology, biopsy and especially otoneurologic imaging. 


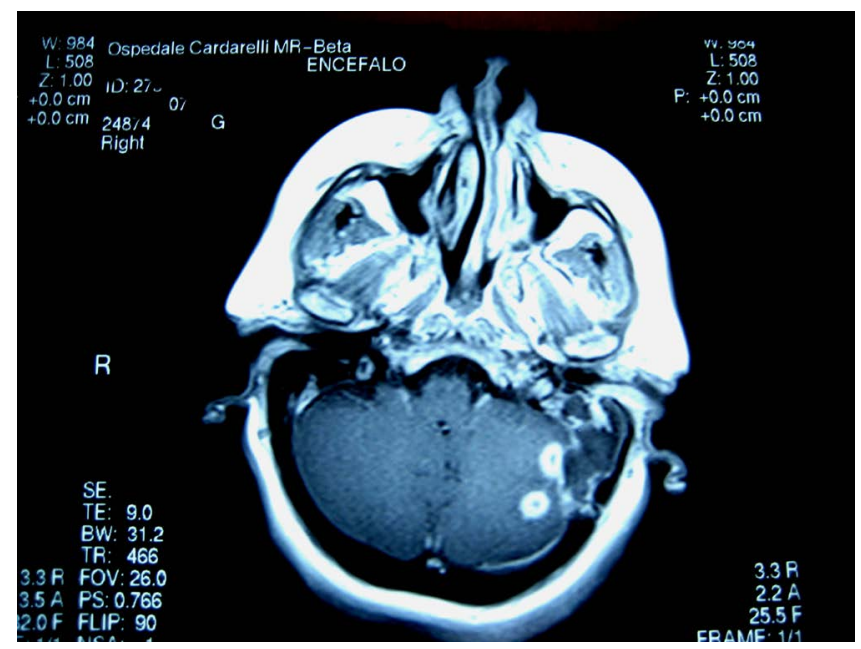

Figure 4. Two nodules in the left cerebellar hemisphere.

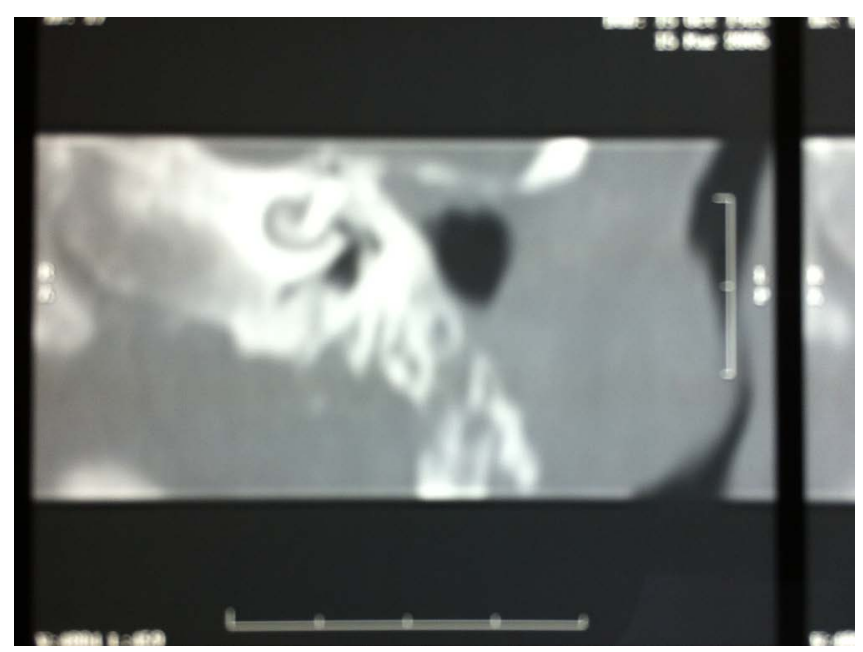

Figure 5. Left ear: mastoidectomy outcomes, soft tissue edema and a pseudo-abscess inferiorly to EAC; material of homogeneous tissue density on new cavum medial wall with second facial tract erosion.

Both patients reported diabetes mellitus type II and medical history of recurrent external otitis.

These clinical features, especially diabetes mellitus presence, confirm classic physiopathology of MEO. In diabetic patients the infection is favored by several factors as chemotaxis reduction, neutrophiles and macrophages phagocytosis decrease, caused also by microangiopathy, duct skin increased $\mathrm{PH}$ and low concentration of lysozyme in earwax.

Clinical examination is fundamental, in particular otomicroscopy; external acoustic canal granulation tissue, especially in osteo-cartilaginous junction could be considered a typical MEO element [5].

Histological examination is necessary for differential diagnosis with other diseases as epithelial malignancies, neuroendocrine tumors, lymphomas and Wegener's granulomatosis [22]; in studied cases, there were not hallmarks, but only non-specific features of acute and chronic inflammation.

Imaging plays a primary role for nosological classification. CT is the gold standard for bone study, it is sensitive to bone erosion. It can identify bone trabecular demineralisation of $30 \%$ or greater, but it is a poor choice to assess treatment response, because of long persistence of these features after disease resolution. It needs to use high-resolution algorithms to avoid misunderstanding of the inflammatory process [23].

MRI is more sensitive than CT [23] in early recognition of soft tissue inflammatory process (fungal infections: 
calcifications, hyperintensity in T2 images or marked hypointensity in the whole sequences because of presence of iron and manganese in fungal elements), intracranial extension, venous sinus thrombosis and skull base involvement.

Angio-MR is considered the gold standard to study extension and involvement of venous sinus [24].

In this study, CT and MRI have premised a good management of cases.

The authors did not use Tc $99 \mathrm{~m}$ or Ga 67 scintigraphy, even if they agree their utility in early diagnosis of MEO.

In this study, antibiotic therapy was based on bacterial culture, despite empiric therapy was the first approach. More effective drugs against Staphylococcus aureus are fluoroquinolones (expecially ciprofloxacin and levofloxacin), that have broad spectrum and good penetration in tissues and bone and teicoplanin effective versus Gram-positive. Third and forth generation cephalosporin (ceftazidime and cefepime), carbapenems (imipenem/ cilastina) and semi-synthetic penicillins (piperacillin/tazobactam) associated to aminoglycosides (tobramycin) are also useful.

Topical medicament provides solution of boric acid in alcohol at $70^{\circ}$. In literature is also reported availment of different topical solution, as Burow solution (aluminum acetate at 13\%), with bacteriostatic activity [25].

Another important feature of antibiotic therapy to consider is its duration. The authors agree on regimens duration from 6 to 8 weeks. This study clarifies as a period of 6 weeks is inadequate for the whole resolution; in fact, the second patient treated with a 6 weeks therapy, presented a disease recurrence after some months. The antibiotic therapy duration is heavily influenced by two different factors: drug-resistence and "adverse drag reactions" (ADR). In this regard it should be noted Shichmanter's study [26] that evaluated ADR appearance in 21 MEO cases. Patients that assumed fluoroquinolones didn't have ADR, while patients treated with other antibiotics (cephalosporins, carbapenems, piperacillin/tazobactam and/or aminoglycoside) had different degrees of ADR including severe neutropenia in two cases. In cases examined in our study there were not ADR, except moderate hypertransaminasemia in the first case and a mild hypoalbuminemia in the second one.

As regard hyperbaric oxygen therapy (HOT), Narozny in 2006 [27] emphasized its important therapeutic role, based on greater provision of oxigen to tissues. The HOT cause edema abatement, fibroblast proliferation, activation of neo-angiogenesis, increased oxygen-dependent bactericidal activity of leukocytes, increased activity of osteoblasts and osteoclasts and enhanced antibacterial activity of many antibiotics. According to Tisch et al. [28] hyperbaric therapy should represent the standard for MEO treatment, despite the difficulty in assessing the effectiveness and usefulness with randomised controlled trials, double-blind, due to the rare incidence of the disease. Despite recognizing the validity, our two patients were not subjected to this therapy.

About surgery role, the authors practiced excision of the granulations from the external auditory canal in both patients; they necessarily performed surgerical drainage of cerebellar abscesses in the first patient, mastoidectomy and mastoidectomy revision in the second one.

\section{Conclusions}

This study underlines that an early diagnosis and adequate therapy can improve the prognosis of a life-threatening infection as MEO.

Specific knowledge of predisposing and causative factors is an important prerequisite for an early identification of clinical manifestations in patients at risk, that should be submitted to specific exams.

Headache not responsive to common anti-inflammatory drugs and possible association with cranial nerve paralytic syndromes, should be immediately predictive of a serious disease process interesting the skull base. Imaging confirms nature and site of osteolytic processes in place.

Among the proposed therapeutic modalities, there is no doubt that the first priority is covered by the first empirical antibiotic treatment and then focused on microbiological and culture results. In cases of MEO by Staphylococcus aureus the authors used ciprofloxacin or levofloxacin for 8 weeks associated with teicoplanin for two/ three weeks.

However, antibiotic therapy is not free from systemic complications and is also serious that it can limit their use and thereby adversely affects the prognosis.

Surgery is necessary in cases of extensive tympanic-mastoid involvement, of facial paralysis "non-responder" and in cases of intracranial implication. The medical and surgical treatment may be associated with successful cycles of hyperbaric oxygen therapy. 


\section{References}

[1] Chandler, J.R. (1968) Malignant External Otitis. Laryngoscope, 78, 1257-1294. http://dx.doi.org/10.1288/00005537-196808000-00002

[2] Hobson, C.E., Moy, J.D., Byers, K.E., Raz, Y., Hirsch, B.E. and McCall, A.A. (2014) Malignant Otitis Externa: Evolving Pathogens and Implications for Diagnosis and Treatment. Otolaryngology—Head and Neck Surgery, 151, 112-116.

[3] Carfrae, M.J. and Kesser, B.W. (2008) Malignant Otitis Externa. Otolaryngologic Clinics of North America, 41, 537549.

[4] Grandis, J.R., Branstetter IV, B.F. and Yu, V.L. (2004) The Changing Face of Malignant (Necrotising) External Otitis: Clinical, Radiological, and Anatomic Correlations. The Lancet Infectious Diseases, 4, 34-39. http://dx.doi.org/10.1016/S1473-3099(03)00858-2

[5] Ophir, H., et al. (2003) Necrotizing (Malignant) External Otitis. American Family Physician, 68, 309-312.

[6] Keay, D.G. and Murray, J.A. (1988) Malignant Otitis Externa Due to Staphylococcus Infection. The Journal of Laryngology \& Otology, 102, 926-927. http://dx.doi.org/10.1017/S0022215100106826

[7] Barrow, H.N. and Levenson, M.J. (1992) Necrotizing “Malignant” External Otitis Caused by Staphylococcus epidermidis. Otolaryngology — Head and Neck Surgery, 118, 94-96. http://dx.doi.org/10.1001/archotol.1992.01880010098023

[8] Soldati, D., Mudry, A. and Monnier, P. (1999) Necrotizing Otitis Externa Caused by Staphylococcus epidermidis. European Archives of Oto-Rhino-Laryngology, 256, 439-441. http://dx.doi.org/10.1007/s004050050184

[9] Thompson, A.C., et al. (2010) Necrotising Otitis Externa: An Unusual Cause of Cranial Nerve Palsy in a Diabetic Haemodialysis Patient. The Journal of the Royal College of Physicians of Edinburgh, 40, 26-28. http://dx.doi.org/10.4997/JRCPE.2010.106

[10] Garcia Rodriguez, J.A., et al. (1992) A Case of Malignant External Otitis Involving Klebsiella oxytoca. The Journal of the Royal College of Physicians of Edinburgh, 11, 75-77. http://dx.doi.org/10.1007/BF01971280

[11] Parize, P., Chandesris, M.O., Lanternier, F., Poirée, S., Viard, J.P., Bienvenu, B., et al. (2009) Antifungal Therapy of Aspergillus fumigatus Invasive Otitis Externa: Efficacy of Voriconazole and Review. Antimicrobial Agents and Chemotherapy, 53, 1048-1053.

[12] Tarazi, A.E., Al-Tawfiq, J.A. and Abdi, R.F. (2012) Fungal Malignant Otitis Externa: Pitfalls, Diagnosis, and Treatment. Otology \& Neurotology, 33, 769-773. http://dx.doi.org/10.1097/MAO.0b013e3182565b46

[13] Clark, J.H., Lin, F.R., Salaria, S.N., Stewart, C.M. and Francis, H.W. (2011) Malignant Otitis Externa Caused by Aspergillus Fumigatus: A Case Report. Otology \& Neurotology, 32, e22-e23. http://dx.doi.org/10.1097/MAO.0b013e3181e3dec7

[14] Loh, S. and Loh, W.S. (2013) Malignant Otitis Externa: An Asian Perspective on Treatment Outcomes and Prognostic Factors. Otolaryngology—Head and Neck Surgery, 148, 991-996. http://dx.doi.org/10.1177/0194599813482107

[15] Franco-Vidal, V., Blanchet, H., Bebear, C., Dutronc, H. and Darrouzet, V. (2007) Necrotizing External Otitis: A Report of 46 Cases. Otology \& Neurotology, 28, 771-773. http://dx.doi.org/10.1097/MAO.0b013e31805153bd

[16] Mani, N., Sudhoff, H., Rajagopal, S., Moffat, D. and Axon, P.R. (2007) Cranial Nerve Involvement in Malignant External Otitis: Implications for Clinical Outcome. Laryngoscope, 117, 907-910.

http://dx.doi.org/10.1097/MLG.0b013e318039b30f

[17] Clerc, N.L., Verillaud, B., Duet, M., Guichard, J., Herman, P. and Kania, R. (2014) Skull Base Osteomyelitis: Incidence of Resistance, Morbidity and Treatment Strategy. Laryngoscope, 124, 2013-2016. http://dx.doi.org/10.1002/lary.24726

[18] Soudry, E., Hamzany, Y., Preis, M., Joshua, B., Hadar, T. and Nageris, B.I. (2011) Malignant External Otitis: Analysis of Severe Cases. Otolaryngology_-Head and Neck Surgery, 144, 758-762. http://dx.doi.org/10.1177/0194599810396132

[19] Chakraborty, D., Bhattacharya, A., Gupta, A.K., Panda, N.K., Das, A. and Mittal, B.R. (2013) Skull Base Osteomyelitis in Otitis Externa: The Utility of Triphasic and Single Photon Emission Computed Tomography/Computed Tomography Bone Scintigraphy. Indian Journal of Nuclear Medicine, 28, 65-69.

[20] Chakraborty, D., Bhattacharya, A., Kamaleshwaran, K.K., Agrawal, K., Gupta, A.K. and Mittal, B.R. (2012) Single Photon Emission Computed Tomography/Computed Tomography of the Skull in Malignant Otitis Externa. American Journal of Otolaryngology, 33, 128-129. http://dx.doi.org/10.1016/j.amjoto.2011.05.002

[21] Sardesai, R.B. and Krishnakumar, T. (2002) Malignant Otitis External-Our Experience. Indian Journal of Otolaryngology and Head \& Neck Surgery, 54, 132-135. 
[22] Bernheim, J. and Sade, J. (1989) Histopathology of the Soft Parts in 50 Patients with Malignant External Otitis. Journal of Laryngology \& Otology, 103, 366-368. http://dx.doi.org/10.1017/S0022215100108977

[23] Karantanas, A.H., Karantzas, G., Katsiva, V., Proikas, K. and Sandris, V. (2003) CT and MRI in Malignant External Otitis: A Report of Four Cases. Computerized Medical Imaging and Graphics, 27, 27-34. http://dx.doi.org/10.1016/S0895-6111(02)00052-6

[24] Yang, T.H., Kuo, S.T. and Young, Y.H. (2006) Necrotizing External Otitis in a Patient Caused by Klebsiela pneumoniae. European Archives of Oto-Rhino-Laryngology and Head \& Neck, 263, 344-346. http://dx.doi.org/10.1007/s00405-005-0998-y

[25] Shimizu, T., Ishinaga, H., Seno, S. and Majima, Y. (2005) Malignant External Otitis: Treatment with Prolonged Usage of Antibiotics and Burow's Solution. Auris Nasus Larynx, 32, 403-406. http://dx.doi.org/10.1016/j.anl.2005.05.010

[26] Shichmanter, R., Miller, E.B. and Landau, Z. (2004) Adverse Drug Reactions Due to Prolonged Therapy for Malignant External Otitis. European Journal of Internal Medicine, 15, 441-445. http://dx.doi.org/10.1016/j.ejim.2004.08.006

[27] Narozny, W., Kuczkowski, J., Stankiewicz, C., Kot, J., Mikaszewski, B. and Przewozny, T. (2006) Value of Hyperbaric Oxygen in Bacterial and Fungal Malignant External Otitis Treatment. European Archives of Oto-Rhino-Laryngology and Head \& Neck, 263, 680-684. http://dx.doi.org/10.1007/s00405-006-0033-y

[28] Tisch, M., Lorenz, K.J., Harm, M., Lampl, L. and Maier, H. (2003) The Treatment of Necrotizing Otitis Externa with a Combination of Surgery, Antibiotics, Specific Immunoglobulins and Hyperbaric Oxygen Therapy. Results of the Ulm Treatment Concept. Journals of Otolaryngology Head and Neck Surgery, 51, 315-320. 
Scientific Research Publishing (SCIRP) is one of the largest Open Access journal publishers. It is currently publishing more than 200 open access, online, peer-reviewed journals covering a wide range of academic disciplines. SCIRP serves the worldwide academic communities and contributes to the progress and application of science with its publication.

Other selected journals from SCIRP are listed as below. Submit your manuscript to us via either submit@scirp.org or Online Submission Portal.
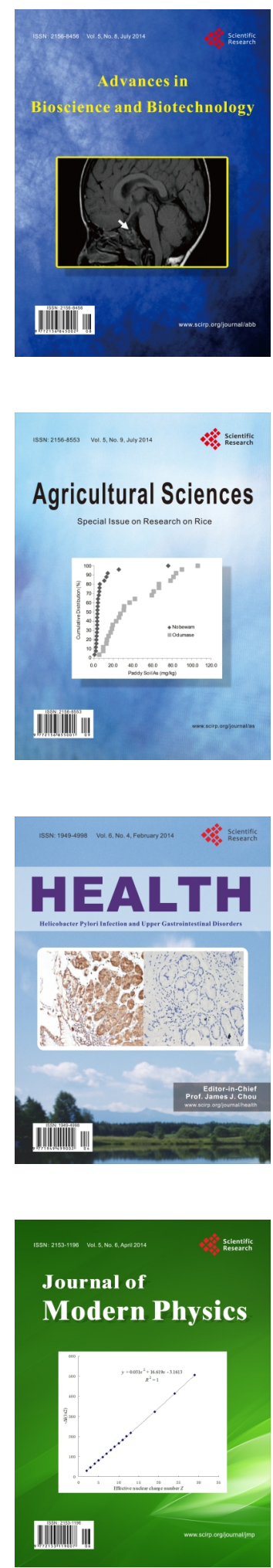
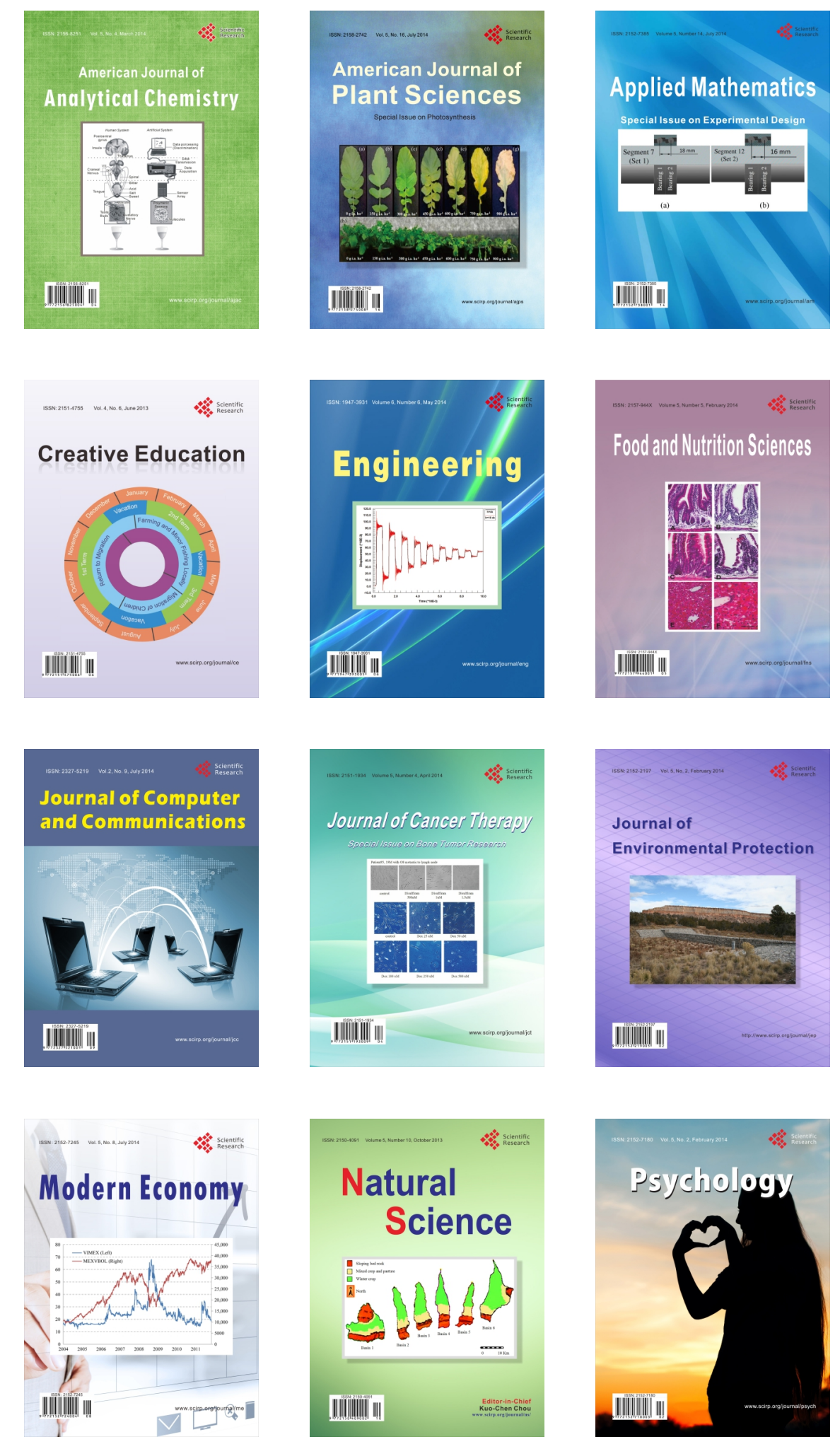